\title{
Conhecimentos de acadêmicos de Medicina de uma instituição de Teresina frente às
}

\section{imunizações}

\author{
Knowledge of medical students from a institution in Teresina facing immunization \\ Conocimientos de estudiantes de medicina de una institución de Teresina frente a la inmunización
}

Recebido: 10/12/2021 | Revisado: 14/12/2021 | Aceito: 17/12/2021 | Publicado: 02/01/2022

Luana Arcoverde de Castro Silveira
ORCID: https://orcid.org/0000-0003-0009-0774
Centro Universitário UNINOVAFAPI, Brasil
E-mail: luanaacsilveira @ gmail.com
Natália França Torres
ORCID: https://orcid.org/0000-0003-2929-3479
Centro Universitário UNINOVAFAPI, Brasil
E-mail: nataliiaf@ hotmail.com
Carlos Gilvan Nunes de Carvalho
ORCID: https://orcid.org/0000-0003-0009-0774
Centro Universitário UNINOVAFAPI, Brasil
E- mail: carlos.carvalho@ @ninovafapi.edu.br

\section{Resumo}

Objetivo: Esse trabalho visou a análise do conhecimento de acadêmicos de medicina sobre as imunizações, a abordagem frente ao paciente acerca da sua atualização vacinal, a atualização da caderneta de vacina de acadêmicos de medicina e o comportamento frente ao seu autocuidado com a imunização. Foi aplicado um questionário com cinco questões que testaram o conhecimento desses estudantes, três avaliaram a prática clínica do acadêmico quanto ao esclarecimento sobre as vacinas para seus pacientes e três questões sobre a atualização vacinal do estudante pesquisado. Resultado: foram coletadas 156 respostas. Observou-se que é restrito o conhecimento acerca das vacinas disponíveis no programa nacional de imunizações visto que muitas vacinas da caderneta da criança não foram assinaladas por muitos estudantes, ao serem questionados sobre as vacinas que recordam ter tomado. Além disso, as cinco questões que analisaram o conhecimento tiveram como resultado índice insatisfatório em 78,8\%. Quando questionados se encaminham o paciente para vacinação dos imunizantes faltantes, apenas $69,9 \%$ afirmam fazer o encaminhamento em pelo menos $50 \%$ das consultas. Viu-se também que $73,1 \%$ dos pesquisados não tem domínio sobre o conteúdo do calendário vacinal de forma a passar informação correta e segura aos pacientes. Um dado positivo coletado foi acerca da confiança na eficácia e importância das vacinas, assertiva que totalizou $91,7 \%$ estudantes que afirmam ter total confiança. Conclusão: O conhecimento sobre as vacinas, a atitude clínica e a atualização vacinal do estudante pesquisado tiveram resultados, em sua maioria, insatisfatórios.

Palavras-chave: Imunização; Conhecimento; Acadêmicos de medicina.

\begin{abstract}
Objective: This work aimed to analyze the knowledge of medical students about immunizations, the approach towards the patient regarding their vaccination update, the updating of the medical students' vaccine booklet and the behavior towards their self-care with immunization. A questionnaire with five questions that tested the knowledge of these students was applied, three assessed the academic's clinical practice regarding the clarification of vaccines for their patients and three questions about the vaccination update of the researched student. Result: 156 responses were collected. It was observed that knowledge about the vaccines available in the national immunization program is limited since many vaccines in the child's booklet were not mentioned by many students, when asked about the vaccines they remember having taken. In addition, the five questions that analyzed knowledge resulted in an unsatisfactory rate of $78.8 \%$. When asked if they refer the patient for vaccination of missing immunization agents, only $69.9 \%$ say they do so in at least $50 \%$ of the consultations. It was also seen that $73.1 \%$ of respondents do not master the content of the vaccination schedule in order to provide correct and safe information to patients. A positive data collected was about confidence in the effectiveness and importance of vaccines, assertion that totaled $91.7 \%$ students who claim to have complete confidence. Conclusion: The researched student's knowledge about vaccines, clinical attitude and vaccination update had unsatisfactory results.
\end{abstract}

Keywords: Immunization; Knowledge; Medical scholars. 


\begin{abstract}
Resumen
Objetivo: Este estudio tuvo como objetivo analizar el conocimiento de los estudiantes de medicina sobre inmunizaciones, el acercamiento al paciente en cuanto a su actualización de vacunación, la actualización del cuadernillo de vacunas de los estudiantes de medicina y el comportamiento hacia su autocuidado con inmunización. Se aplicó un cuestionario con cinco preguntas que ponían a prueba los conocimientos de estos estudiantes, tres evaluaban la práctica clínica del académico en cuanto a la aclaración de vacunas para sus pacientes y tres preguntas sobre la actualización de vacunación del estudiante investigado. Resultado: se recopilaron 156 respuestas. Se observó que el conocimiento sobre las vacunas disponibles en el programa nacional de inmunización es limitado ya que muchas vacunas en el cuadernillo del niño no fueron mencionadas por muchos estudiantes, cuando se les preguntó sobre las vacunas que recuerdan haber tomado. Además, las cinco preguntas que analizaron el conocimiento arrojaron una tasa insatisfactoria del 78,8\%. Cuando se les pregunta si derivan al paciente para vacunación de inmunizadores faltantes, solo el 69,9\% dice que lo hace en al menos el 50\% de las consultas. También se observó que el 73,1\% de los encuestados no domina el contenido del calendario de vacunación para brindar información correcta y segura a los pacientes. Un dato positivo recogido fue sobre la confianza en la eficacia y la importancia de las vacunas, afirmación que totalizó el $91,7 \%$ de los estudiantes que aseguran tener total confianza. Conclusión: El conocimiento del estudiante investigado sobre vacunas, actitud clínica y actualización de vacunación arrojó resultados insatisfactorios.
\end{abstract}

Palabras clave: Inmunización; Conocimiento; Eruditos médicos.

\title{
1. Introdução
}

Em 1973 foi formulado o Programa Nacional de Imunizações - PNI, por determinação do Ministério da Saúde, com o objetivo de coordenar as ações de imunizações que se caracterizavam, até então, pela descontinuidade, pelo caráter episódico e pela reduzida área de cobertura (Chehuen Neto et al., 2010; Yassi et al., 2016; Canadian-South African partnership of partnerships, 2020).

Ao longo do tempo, a atuação do PNI, ao consolidar uma estratégia de âmbito nacional, apresentou avanços. As metas mais recentes contemplam erradicação do sarampo e a eliminação do tétano neonatal além do controle de outras doenças imunopreveníveis como Difteria, Coqueluche e Tétano acidental, Hepatite B, Meningites, Febre Amarela, formas graves da Tuberculose, Rubéola e Caxumba em alguns Estados, bem como, a manutenção da erradicação da Poliomielite (Sato et al., 2015).

As vacinas combatem as consequências devastadoras que as doenças infecciosas impuseram à humanidade por séculos, representam o investimento em saúde com melhor custo-efetividade. Mas, para isso, é necessário que sejam aceitas, obtenham a confiança do público alvo e atinjam amplo e adequado uso (Hesse et al., 2020; Nóvoa et al., 2020).

Nesse contexto a imunização oferece imensos benefícios para a população, sendo uma das grandes conquistas da ciência e da saúde pública durante o século 20 isso porque, as vacinas são responsáveis por reduzir uma variedade de doenças infecciosas antes comuns e mortais historicamente. (Deus et al., 2016; Approved by the National Vaccine, 2020).

Nesse âmbito, surge o papel dos estudantes da área da saúde e profissionais de saúde, em disseminar informações sobre a importância e efetividade da imunização para assegurar a saúde da humanidade. Entretanto, para que o estudante de medicina possa exercer seu papel como contribuinte da efetivação da atenção primária, é preciso que tenham um vasto conhecimento acerca das imunizações, para que possam avaliar de forma correta os seus pacientes (Júnior et al., 2013; de Carvalho et al., 2020).

Porém, mesmo com toda a disponibilidade do PNI, que garante a distribuição e a aplicação das vacinas, com vistas a benefícios individuais e coletivos, pessoas e grupos que declaram preocupações com a segurança e a eficácia das vacinas ocorrem no Brasil e em todo o mundo (Ferreira Silva-junior et al., 2014; de Carvalho et al., 2020).

Com a crescente disponibilidade de novas vacinas e as frequentes atualizações dos calendários vacinais, manter-se atualizado e conhecer as indicações, precauções e possibilidade de eventos adversos são constantes desafios para esses profissionais. Além disso, considerando-se sua exposição ocupacional, estão expostos a adquirir e transmitir doenças infecciosas, o que exige que mantenham em dia a sua própria situação vacinal. O profissional e estudante da saúde deve estar 
Research, Society and Development, v. 11, n. 1, e57111240603, 2022

(CC BY 4.0) | ISSN 2525-3409 | DOI: http://dx.doi.org/10.33448/rsd-v11i1.24603

munido de conhecimento técnico e habilidade de comunicação, aproveitando cada oportunidade para esclarecer aos pacientes sobre o assunto da vacinação, assegurando-os confiança nas vacinas (Cometto et al., 2020).

Assim, atualizações frequentes sobre vacinas disponíveis, mudanças nos calendários vacinais, ocorrência e manejo dos eventos adversos são indispensáveis. Tal atualização pode ser feita por meio de cursos, documentos científicos e consultas com especialistas, permitindo ao médico responder as questões apresentadas com segurança e credibilidade.

Diante do exposto a vacinação do profissional de saúde, seus conhecimentos sobre o assunto e a sua própria confiança nas vacinas são necessários para orientar sua conduta na indicação dos imunizantes aos pacientes por ele assistidos (Maia et al., 2020). Dessa forma a presente pesquisa teve como objetivo verificar os conhecimentos de acadêmicos de Medicina de uma Instituição de Teresina frente às imunizações.

\section{Metodologia}

Trata-se de um estudo descritivo transversal com abordagem quantitativa, cuja coleta de dados foi realizada no período de 1 de agosto a 30 de outubro de 2021 através da plataforma Google Forms ${ }^{\circledR}$. A coleta ocorreu após a aprovação pelo Comitê de Ética em Pesquisa do UNINOVAFAPI com o número do CAAE 45462221.3.0000.5210 em 01 de junho de 2021 em consonância com a Resolução 466/2012 do Conselho Nacional de Saúde e todas as resoluções vigentes no Brasil em relação à ética da pesquisa científica.

Um estudo transversal é definido como um tipo de pesquisa observacional que analisa dados de variáveis coletadas em um determinado ponto no tempo em uma população de amostra ou um subconjunto predefinido. Embora a pesquisa transversal não envolva a realização de experimentos, os pesquisadores costumam usá-la para compreender os resultados nas ciências físicas e sociais e em muitos setores de negócios (Severino, 2017).

O estudo contemplou 156 estudantes de medicina selecionados, aleatoriamente, entre os entre os 232, matriculados na UNINOVAFAPI, no $8^{\circ}, 9^{\circ}$ e $10^{\circ}$ períodos no primeiro semestre de 2021 que aceitaram participar da pesquisa concordando com o Termo de Consentimento Livre e Esclarecido (TCLE) e responderam o questionário completamente.

Os alunos foram convidados a participar da pesquisa por meio de um link do formulário encaminhado pelo líder de turma nos grupos de Whatsapp das respectivas turmas. O questionário foi anônimo, não se teve contato com o e-mail do participante e não houveram campos para identificação do acadêmico, além disso todas as questões foram objetivas.

O questionário foi elaborado pelos pesquisadores e contém apenas questões objetivas. Neste, os acadêmicos foram inquiridos sobre os seus conhecimentos em relação as vacinas administradas nas respectivas situações solicitadas, conhecimentos sobre as vacinas do PNI e suas restrições e indicações e conduta pós exposição a doença imunoprevinível. Em seguida, foram questionados sobre a atitude clínica quanto às imunizações: Se verifica o cartão vacinal nas consultas ambulatoriais rotineiramente, se ao atender um paciente que não sabe informar suas vacinas, ou que perdeu o cartão de vacina, encaminha para a atualização vacinal, se sente que tem domínio sobre o calendário vacinal de forma a passar informação, credibilidade e segurança na eficácia das vacinas para os pacientes. Por fim, os acadêmicos serão avaliados sobre o seu cartão vacinal, se tem confiança na eficácia e importância das vacinas, se tem cartão de vacina e costuma atualizá-lo, quais vacinas recorda ter tomado e quais vacinas precisaria tomar para manter o cartão vacinal atualizado na idade atual.

Foi atribuído um valor máximo de 1 ponto para cada resposta correta e zero para as respostas erradas às questões de conhecimento (questões 1 a 5). O total de pontos foi 5 que correspondeu a 100\%, equivalente ao acerto de 5 questões que possuem o mesmo peso 1 . Posteriormente foi aplicada uma escala categorizando-os em intervalos percentuais de duas classes: insatisfatório de 0 a 50\% (0 a 2 pontos); regular de 50 a 75\% (3 pontos) e satisfatório de 75 a 100\% (4 a 5 pontos) de acertos, entende-se por satisfatório como sinônimo de rendimento suficiente, que foi alcançado o resultado esperado. 
Os dados coletados serão submetidos a uma análise estatística descritiva através das frequências absolutas e relativas. O processamento será feito por meio da planilha Excel e do Programa SPSS. Os resultados serão apresentados em forma de tabelas e gráficos.

\section{Resultados e Discussão}

Dentre os 156 participantes que aceitaram participar da pesquisa, 47 eram do décimo, 39 do nono e 70 do oitavo período. Analisando os dados coletados foi obtido um resultado insatisfatório quanto ao conhecimento dos estudantes de medicina sobre as imunizações.

Das 5 questões que analisaram o conhecimento desses estudantes (Tabela 1), a primeira avaliou a conduta frente à vacina da gripe no caso de desconhecimento sobre a vacinação do paciente, tendo sido obtido $18,6 \%$ de acertos. A segunda analisou a conduta frente a um acidente com material possivelmente contaminado pelo vírus do tétano, sendo obtido $17,3 \%$ de acertos. A terceira questão indagou sobre quais vacinas são feitas aos 12 meses de idade e a via de administração, com $26,3 \%$ de acertos. A quarta questão avaliou o conhecimento sobre a composição da vacina pentavalente, com 44,9\% de acertos. A quinta questão pediu a afirmativa correta em relação ao calendário nacional de vacinação do ministério da saúde, a resposta correta continha que a vacina Meningocócica $\mathrm{C}$ tem como esquema vacinal duas doses (3 meses e 5 meses) com reforço aos 12 meses, foi obtido $51,3 \%$ de acertos. Conclui-se que a taxa de acertos foi muito baixa e insatisfatória, o que chama atenção para a necessidade de maior inclusão deste conteúdo no currículo médico.

Tabela 1: Acertos por questão.

\begin{tabular}{|c|c|c|c|}
\hline & & Frequência & $\%$ \\
\hline \multirow{5}{*}{ Questões } & $\begin{array}{l}\text { CONDUTA FRENTE À VACINA DA GRIPE NO CASO DE } \\
\text { DESCONHECIMENTO SOBRE A VACINAÇÃO DO PACIENTE }\end{array}$ & 29 & $18,6 \%$ \\
\hline & $\begin{array}{l}\text { CONDUTA FRENTE A UM ACIDENTE COM MATERIAL } \\
\text { PÉRFURO-CORTANTE POSSIVELMENTE CONTAMINADO PELO } \\
\text { VÍRUS DO TÉTANO }\end{array}$ & 27 & $17,3 \%$ \\
\hline & $\begin{array}{l}\text { VACINAS OFERTADAS EM DETERMINADA IDADE E A VIA DE } \\
\text { ADMINISTRAÇÃO }\end{array}$ & 41 & $26,3 \%$ \\
\hline & CONHECIMENTO SOBRE A COMPOSIÇÃO DE VACINAS & 70 & $44,9 \%$ \\
\hline & $\begin{array}{l}\text { CONHECIMENTO SOBRE O CALENDÁRIO NACIONAL DE } \\
\text { VACINAÇÃO DO MINISTÉRIO DA SAÚDE E O ESQUEMA } \\
\text { VACINAL DE UMA DETERMINADA VACINA }\end{array}$ & 80 & $51,3 \%$ \\
\hline
\end{tabular}

Fonte: Autores.

Ao analisar o número de acertos por estudante pesquisado e classificar como insatisfatório, regular ou satisfatório (Tabelas 2 e 3), verificou-se que o resultado foi em sua maior parte insatisfatório, totalizando 78,8\% dos casos. 16,7\% dos alunos tiveram um desempenho regular, e apenas 4,5\% foram classificados como conhecimento satisfatório. 
Tabela 2: Pontos obtidos por participante.

\begin{tabular}{ll|c|c}
\hline & & Frequência & $\%$ \\
\hline & 0 & 34 & $21,8 \%$ \\
Quantidade de Pontos & 2 & 39 & $25,0 \%$ \\
& 3 & 50 & $32,1 \%$ \\
& 4 & 26 & $16,7 \%$ \\
& 5 & 5 & $3,2 \%$ \\
& 5 & 2 & $1,3 \%$ \\
\hline
\end{tabular}

Fonte: Autores.

Tabela 3: Classificação.

\begin{tabular}{ll|c|c}
\hline & & Frequência & $\%$ \\
\hline \multirow{4}{*}{ Classificação } & INSATISFATÓRI & 123 & $78,8 \%$ \\
& O & & \\
& REGULAR & 26 & $16,7 \%$ \\
& SATISFATÓRIO & 7 & $4,5 \%$ \\
\hline
\end{tabular}

Fonte: Autores.

Nas questões que avaliaram a atitude clínica quanto às imunizações, $31 \%$ dos participantes pesquisados afirmam fazer a verificação do cartão vacinal, quase sempre, pelo menos $50 \%$ das vezes. Enquanto que 39,7\% não fazem a verificação do cartão vacinal. Logo, conclui-se que a maior parte dos estudantes pesquisados não verificam o cartão vacinal ou não o fazem de rotina, sendo considerado insatisfatório levando em conta a importância dessa atitude na erradicação de doenças imunopreviníveis.

Após avaliarem um paciente que perdeu o cartão de vacina ou que não sabe informar se vacinou ou não contra determinada doença, o estudante foi questionado se realiza ou não o encaminhamento para atualização vacinal. Verificou-se resultado insatisfatório visto que apenas $69,9 \%$ encaminham o paciente para atualizar o cartão vacinal em pelo menos $50 \%$ das consultas, enquanto que 30,1\% não encaminham, representando uma lacuna preocupante na atenção primária dos hospitais escola.

Constatou-se que 73,1\% dos pesquisados, na maioria dos casos, não tem domínio sobre o calendário vacinal de forma a passar informação, credibilidade e segurança na eficácia das vacinas para seus pacientes. $23,7 \%$ diz não tem domínio nenhum. Esse dado, ratifica o desconhecimento dos estudantes sobre o calendário vacinal, já avaliado pelas questões deste trabalho, que mediram o conhecimento, além disso, resultado semelhante foi obtido em uma pesquisa publicada em 2020, na qual o questionário aplicado por eles para avaliação do conhecimento geral sobre vacinas, teve como resultado 59,5\% de conhecimento insatisfatório, 37,6\% satisfatório e apenas 2,8\% muito satisfatório, baseando-se nos critérios de número de acertos da pesquisa (De Carvalho et al., 2020).

Quanto às imunizações do estudante pesquisado, 91,7\% tem total confiança na eficácia e importância das vacinas enquanto que $6,4 \%$ tem receio pelos efeitos adversos, dado importante que reverbera de forma positiva para os pacientes assistidos por esses profissionais. Ao ser questionado sobre a atualização do seu cartão vacinal $71,8 \%$ dos pesquisados afirma 
Research, Society and Development, v. 11, n. 1, e57111240603, 2022

(CC BY 4.0) | ISSN 2525-3409 | DOI: http://dx.doi.org/10.33448/rsd-v11i1.24603

ter seu cartão atualizado. Resultado insatisfatório visto que o estudante de medicina está em constante contato com risco biológico, sendo essencial sua proteção individual, e os $28,2 \%$ que dizem não ter ou não atualizar seu cartão vacinal devem ser alertados para os riscos aos quais estão expostos, além do risco de disseminação em cadeia de doenças imunopreviníveis. Esse dado coletado se assemelha ao que visualizou Cabrera et al (2011), na qual, dos alunos pesquisados, 73,8\% referem ter o cartão de vacina atualizado enquanto que 26,2\% não estavam com o cartão atualizado. Esses dados devem ser considerados, visto que os acadêmicos e profissionais da área da saúde, possuem risco aumentado de contraírem doenças infecciosas devido ao contato rotineiro com indivíduos e ambientes infectados (de Mello; Albuquerque; Campos, 2020).

Ao questionar os estudantes sobre as vacinas que recorda ter tomado, viu-se que $95,5 \%$ informam ter vacinado contra a tuberculose e 96,8\% informam ter tomado a primeira dose contra Hepatite B ainda na infância, o que se mostrou satisfatório por ter sido mais de $90 \%$ da amostra pesquisada, correspondente à meta definida pelo Ministério da Saúde, de $90 \%$ para a vacina BCG (da Tuberculose) e 95\% para as demais vacinas (de Mello; Albuquerque; Campos, 2020).

No entanto, apenas 23,7\% recordam ter tomado a vacina contra o HPV, que tem um fator limitante por só ser disponível na rede pública para menores de 15 anos ou pacientes soropositivos/imunodeprimidos, o que pode não ter abrangido a maioria dos pesquisados, acadêmicos com idade média de 20 anos, sendo necessária a vacinação com recursos próprios. As outras vacinas presentes no calendário vacinal do ministério da saúde foram pouco assinaladas pelos estudantes, constatandose o desconhecimento sobre sua própria situação vacinal. Tal fato evidencia uma falha nos programas de prevenção primária das universidades, de grande alerta principalmente por se tratar de um público tão exposto, principalmente nos últimos períodos do curso que dizem respeito ao internato, quando o contato com ambientes possivelmente contaminados é maior.

Devido à diminuição da circulação de várias doenças evitáveis por vacinas, algumas pessoas realmente acreditam que a prevenção é secundária ou mesmo desnecessária. Desde 2012, a OMS reafirma a importância desse tema e suas implicações, buscando compreender esse fenômeno e reunindo evidências para desenvolver intervenções de saúde pública para reverter essa tendência. A hesitação vacinal é definida como o atraso no calendário vacinal ou a recusa em receber as vacinas recomendadas, apesar da disponibilidade nos serviços de saúde (de Souza et al., 2018; Mizuta et al., 2019; Williams et al., 2020).

No caso da Hepatite B e tétano, apenas 70,5\% tem ciência de ter concluído o esquema vacinal com a Pentavalente, e 73,1\% afirma ter tomado os dois reforços de DTP. Essa situação é preocupante pois no ambiente de trabalho, o risco de infecção está associado a acidente com material perfuro cortante e ao não uso dos equipamentos de proteção individual, meios pelos quais a contaminação pela Hepatite B e tétano podem se estabelecer. Um dado relevante que reforça esse risco é que segundo a Organização Mundial da Saúde, mais de 1,4 milhões de pessoas no mundo sofrem de complicações relacionadas à saúde ocupacional e ainda na década de 70 foram registrados 4.468 acidentes de trabalho em hospitais do Brasil (Silva Junior et al., 2013).

Segundo Mizuta et al (2018) a hesitação vacinal da população, pode estar relacionada ao conhecimento insuficiente dos profissionais de saúde que atuam nos serviços de vacinação. O calendário vacinal brasileiro tornou-se mais complexo, exigindo amplo conhecimento dos profissionais de saúde sobre as vacinas e suas atualizações, principalmente para as crianças que chegam tarde aos serviços de vacinação. Com o objetivo de capacitar as equipes, o PNI tem investido em treinamentos online para aumentar a capilaridade da rede, com diversos cursos oferecidos nesta plataforma, abordando diversos temas por meio de metodologias diversas (Silva Junior et al., 2013). Tal fato contribui para alavancar a confiança na vacina, e reestabelecer a busca pela imunização na população geral.

Sobre as imunizações contra sarampo, caxumba e rubéola, apenas 73,7\% dos pesquisados tem ciência de ter tomado a primeira dose do imunizante, e 70,5\% de ter tomado a segunda dose, logo só podemos afirmar com certeza que apenas $70,5 \%$ tem proteção contra esses vírus. No estudo de Mello; Albuquerque; Campos (2020) ao questionar sobre o esquema vacinal dos alunos entrevistados, o imunizante para esses três vírus, a tríplice viral, estava presente na caderneta de $80,9 \%$ dos pesquisados. 
Quanto à vacina contra a Febre Amarela, 85,9\% dos estudantes recordam ter tomado, e 57,7\% dizem ter tomado o reforço, fazendo um paralelo com o estudo feito por de Mello; Albuquerque; Campos (2020) em que apenas 40,1\% dos estudantes referem ter tomado esta vacina.

Sabe-se que no Brasil o Programa Nacional de Imunização (PNI) é o órgão responsável pelas vacinações, auxiliando no controle de muitas doenças endêmicas. No entanto, nos últimos anos, parte significativa da sociedade aderiu ao movimento anti-vacina, comprometendo a segurança de toda sociedade (De Carvalho et al., 2020). Dentre algumas vacinas que vem sendo negligenciadas podemos citar a Febre Amarela, a Tuberculose e o Sarampo, sendo este último motivo de grande preocupação devido ao aumento abrupto no número de casos nos anos de 2018 e 2019 em todo território nacional (Nóvoa et al., 2020). Em contrapartida a esse dado, a vacina contra a Tuberculose, no presente estudo, teve resultado satisfatório quanto à presença desta no calendário vacinal dos estudantes da pesquisa.

De acordo com Deus et al (2016) é necessário ampliar ainda a formação dos profissionais de saúde nas salas de aula. Essa deve ser uma prioridade não só das universidades como do Ministério da Saúde, mas também dos estados e municípios, que são responsáveis por manter suas redes preparadas para atender a população.

Estudos mostram que a recomendação por profissionais de saúde é um fator determinante para a aceitação da vacina. O papel do médico tem sido apontado como um dos principais facilitadores para a adesão à vacinação, principalmente para as vacinas às quais a população apresenta alguma resistência, como o HPV. Profissionais qualificados que saibam indicar as vacinas e esclarecer as dúvidas da população são, portanto, uma estratégia importante para aumentar a cobertura vacina (Silva Junior et al., 2013; De Carvalho et al., 2020).

Outro fator relacionado ao aumento da hesitação vacinal em profissionais da saúde caracteriza-se por um aumento da propagação de notícias falsas nas redes sociais. Frases de efeito e de apelo emocional, sem evidências científicas, amplamente compartilhadas nas redes sociais e aplicativos de mensagens, acabam por confundir e espalhar possíveis efeitos colaterais. $\mathrm{O}$ Ministério da Saúde brasileiro identificou as notícias falsas como um dos motivos para a redução das taxas de imunização no Brasil. De acordo com pesquisa do Ministério da Saúde do Brasil nas redes sociais em 2018, 89\% das notícias falsas relacionadas à saúde atacavam especificamente a credibilidade das vacinas. (De Carvalho et al., 2020; Nóvoa et al., 2020; Gadelha et al., 2020).

A superação dos desafios exige uma integração crescente do PNI em todos os níveis administrativos do SUS, bem como nas demais áreas do setor saúde e nos setores socioeducativos e, principalmente, a plena participação dos trabalhadores da saúde que disponibilizam a vacinação para toda a população, para que isso ocorra faz-se necessário o conhecimento de estudantes a respeito de tal questão, visando que estes profissionais possam, não se previnir de possíveis infecções como também utilizar tais conhecimentos com a população geral (Nóvoa et al., 2020).

\section{Conclusão}

A conscientização dos acadêmicos de medicina sobre saúde ocupacional e a importância do esquema vacinal completo é necessária, o que resultará na redução da transmissão de várias doenças, visto que estes têm contato constante com indivíduos e locais contaminados, sendo potenciais disseminadores de doenças infectocontagiosas.

Além disso, a prevenção primária proporcionada pela vacinação tem sua eficácia potencializada se o profissional médico, a partir da sua vivência acadêmica, tiver conhecimento sobre o assunto para abordar corretamente em suas condutas médicas.

Esse trabalho pôde concluir que é necessário o maior comprometimento dos estudantes com sua imunização, além da necessidade de voltarem mais a atenção para o domínio do conteúdo do calendário vacinal do Ministério da Saúde. 


\section{Referências}

Almeida, A. A., Neves, B. R., Palhares, F. R. D., Raposo, F. B. C., Handere, M. P., de Oliveira Freitas, Y., \& do Carmo Cupertino, M. (2021). Vacinação dos estudantes de medicina e o papel das instituições de ensino superior na prevenção primária. Revista de Medicina, $100(2), 112-118$.

Approved by the National Vaccine Advisory Committee on September 17, 2019. (2020). 2020 National Vaccine Plan Development: Recommendations From the National Vaccine Advisory Committee. Public Health Reports, 135(2), 181-188.

Cabrera, E. M. S., \& Merege, C. E. D. S. (2011). Inquérito vacinal de alunos da graduação em medicina e enfermagem da Faculdade de Medicina de São José do Rio Preto (SP, Brasil) nos anos de 2006 e 2007 e suas possíveis implicações na atuação discente. Ciência \& Saúde Coletiva, 16, $547-552$.

Canadian-South African partnership of partnerships. Globalization and health, 12(1), 1-15.

Chehuen Neto, J. A., Sirimarco, M. T., Leite, I. C. G., Gonçalves, M. P. C., Delgado, Á. A. D. A.,

Camilo, G. B., \& Abreu, N. A. D. (2010). Situação vacinal dos discentes da Faculdade de Medicina da UFJF-MG. Revista Brasileira de Educação Médica, 34, 270-277.

Cita, P. E. (2020). Application of Lean Distribution in National Vaccine Program of Indonesia: Case Study in Banten Province. International Proceedings The 2nd ISMoHIM 2020.

Cometto, G., Buchan, J., \& Dussault, G. (2020). Developing the health workforce for universal health coverage. Bulletin of the World Health Organization, 98(2), 109.

de Carvalho, P. É. F. R., de Carvalho Nogueira, E. F., dos Santos, C. S., de Freitas Coelho, L., Hande, P., Moura, V. H. N., \& da Fonseca Lima, E. J. (2020). Conhecimento sobre imunização entre os estudantes de medicina de uma Escola médica de Recife. Research, Society and Development, 9(11), e67191110542e67191110542.

Mello, V. M. O., Albuquerque, V. L. D. S. P., \& Campos, J. S. (2020). Situação vacinal de acadêmicos de medicina e sua percepção sobre a vacinação.

Souza, G. L., Von Randow, R. M., \& da Silva, J. S. (2019). Realidade do cartão de vacina de alunos de cursos da saúde: uma ação preventiva. Anais do Simpósio de Enfermagem, 1(1).

Deus, S. R. M. D., Marques, A. D. B., Texeira, J. C. L., Deus, P. R. M. D., Moraes, M. E. A. D., \& Macêdo, D. S. (2016). Estudo dos procedimentos quanto à conservação das vacinas do Programa Nacional de Imunização. Rev. enferm. UFPE on line, 1038-1046.

Ferreira Silva-junior, M., Freire de Assis, R. I., Rangel Gomes, C. L., Vitali Miclos, P., de Sousa, H. A., \& José Gomes, M. (2014). Conhecimento atual sobre a necessidade de imunização da hepatite B dos acadêmicos da área da saúde de uma universidade brasileira. Archives of Dental Science/Arquivos em Odontologia, 50(3)

Gadelha, C. A. G. (2020). Programa Nacional de Imunizações: o desafio do acesso universal no Século XXI.

Hesse, E. M., Atanasoff, S., Hibbs, B. F., Adegoke, O. J., Ng, C., Marquez, P., \& Nair, N. (2020). Shoulder injury related to vaccine administration (SIRVA): petitioner claims to the National Vaccine Injury Compensation Program, 2010-2016. Vaccine, 38(5), 1076-1083.

Júnior, M. F. S. S., de Assis, R. I. F., de Sousa, H. A., Miclos, P. V., \& Gomes, M. J. (2013). Conhecimento dos acadêmicos de odontologia da Ufes sobre a necessidade de imunização. Revista Brasileira de Pesquisa em Saúde/Brazilian Journal of Health Research.

Maia, M. D. L. D. S., Oliveira, P. M. N. D., Brum, R. C., Lignani, L. K., \& Figueira, J. T. D. O. (2020). Pesquisa clínica para o Programa Nacional de Imunizações. Cadernos de Saúde Pública, 36, e00182719.

Mizuta, A. H., Succi, G. D. M., Montalli, V. A. M., \& Succi, R. C. D. M. (2018). Percepções acerca da importância das vacinas e da recusa vacinal numa escola de medicina. Revista Paulista de Pediatria, 37, 34-40.

National Vaccine Advisory Committee. (2017). Evaluation of the 2010 National Vaccine Plan mid-course review: recommendations from the National Vaccine Advisory Committee. Public Health Reports (1974), 132(4), 411-430.

Nóvoa, T. D. A., Cordovil, V. R., Pantoja, G. M., Ribeiro, M. E. S., dos Santos Cunha, A. C., Benjamin, A. I. M., ... \& Santos, F. A. (2020). Cobertura vacinal do programa nacional de imunizações (PNI). Brazilian Journal of Health Review, 3(4), 7863-7873.

Sato, A. P. S. (2015). Programa Nacional de Imunização: Sistema Informatizado como opção a novos desafios. Revista de Saúde Pública, 49.

Silva Junior, J. B. D. (2013). 40 anos do Programa Nacional de Imunizações: uma conquista da Saúde Pública brasileira. Epidemiologia e Serviços de Saúde, 22(1), 7-8.

Severino, A. J. (2017). Metodologia do trabalho científico. Cortez editora.

Silva, A. A. D., Teixeira, A. M. D. S., Domingues, C. M. A. S., Braz, R. M., \& Cabral, C. M. (2021). Avaliação do Sistema de Vigilância do Programa Nacional de Imunizações-Módulo Registro do Vacinado, Brasil, 2017. Epidemiologia e Serviços de Saúde, 30.

Williams, S. R., LeBuhn, H. M., Driscoll, A. J., Neuzil, K. M., Chen, W. H., \& Ortiz, J. R. (2020). Which countries have adult vaccine programs? A global review of national adult influenza and pneumococcal vaccine policies. A59. clinical diagnosis, prediction and outcomes of lung infections, A2146-A2146.

Yassi, A., Zungu, M., Spiegel, J. M., Kistnasamy, B., Lockhart, K., Jones, D., \& Darwin, L. (2016). Protecting health workers from infectious disease transmission: an exploration of a Canadian-South African partnership of partnerships. Globalization and health, 12(1), 1-15. 